\title{
Mechanical performance of encapsulated restorative glass-ionomer cements for use with Atraumatic Restorative Treatment (ART)
}

\author{
Gustavo Fabián MOLINA ${ }^{1}$, Ricardo Juan CABRAL ${ }^{2}$, Ignacio MAZZOLA ${ }^{3}$, Laura BRAIN LASCANO ${ }^{3}$, Jo. E. FRENCKEN $^{4}$ \\ 1- DDS, Associate Professor, Department of Dental Materials, School of Dentistry, National University of Córdoba, Córdoba, Argentina. \\ 2- DDS, PhD, Head Professor, Department of Dental Materials, School of Dentistry, National University of Córdoba, Córdoba, Argentina \\ 3- DDS, Assistant Professor, Department of Dental Materials, School of Dentistry, National University of Córdoba, Córdoba, Argentina. \\ 4- DDS, MSc, PhD. Associate Professor, Department of Global Oral Health, School of Dental Sciences, Radboud University, Nijmegen Medical Centre, \\ Nijmegen, The Netherlands.
}

Corresponding address: Gustavo Fabián Molina - Cátedra de Materiales Dentales, Facultad de Odontología - Universidad Nacional de Córdoba, Argentina - Av. Maipú 177 4 B - 5000 - Córdoba - Argentina - Phone/Fax.: 00543514221355 - e-mail: gfmolina@hotmail.com

Received: January 28, 2013 - Modified: March 30, 2013 - Accepted: April 9, 2013

\section{ABSTRACT}

\begin{abstract}
$T^{n}$ he Atraumatic Restorative Treatment (ART) approach was suggested to be a suitable method to treat enamel and dentine carious lesions in patients with disabilities. The use of a restorative glass-ionomer with optimal mechanical properties is, therefore, very important. Objective: To test the null-hypotheses that no difference in diametral tensile, compressive and flexural strengths exists between: (1) The EQUIA system and (2) The Chemfil Rock (encapsulated glass-ionomers; test materials) and the Fuji 9 Gold Label and the Ketac Molar Easymix (hand-mixed conventional glass-ionomers; control materials); (3) The EQUIA system and Chemfil Rock. Material and Methods: Specimens for testing flexural $(n=240)$ and diametral tensile $(n=80)$ strengths were prepared according to standardized specifications; the compressive strength $(n=80)$ was measured using a tooth-model of a class II ART restoration. ANOVA and Tukey $B$ tests were used to test for significant differences between dependent and independent variables. Results: The EQUIA system and Chemfil Rock had significantly higher mean scores for all the three strength variables than the Fuji 9 Gold Label and Ketac Molar Easymix $(\alpha=0.05)$. The EQUIA system had significant higher mean scores for diametral tensile and flexural strengths than the Chemfil Rock $(\alpha=0.05)$. Conclusion: The two encapsulated high-viscosity glass-ionomers had significantly higher test values for diametral tensile, flexural and compressive strengths than the commonly used hand-mixed high-viscosity glass-ionomers.
\end{abstract}

Key words: Dentistry. Dental Atraumatic Restorative Treatment. Coatings. Glass ionomer cements. Physical properties.

\section{INTRODUCTION}

Managing dentine carious lesions in patients with physical and/or intellectual disabilities represents a challenge. These populations present characteristics that are difficult to deal with through application of conventional restorative treatment approaches using rotary driven equipment. Problems include: poor cooperation during dental procedures because the patients cannot remain motionless; difficulty in keeping their mouths open constantly while a restorative procedure is performed; difficulties in accessing cavities and gaining acceptance of rotary instrumentation, mainly because of the noise; strong grinding of teeth and severe drooling ${ }^{16,19}$.

In addition to being a well-accepted dental caries management approach in children ${ }^{2,10}$, the Atraumatic Restorative Treatment (ART) has been recommended as a possible treatment protocol for use amongst people with disabilities ${ }^{12,17}$. It was thought that ART could overcome some of the problems mentioned above in treating people with disabilities, because it only uses hand instruments, which are not noisy. Anecdotal information confirms 
the above to be correct. However, the evidence for the effectiveness of ART in people with disabilities is very weak ${ }^{17}$. If ART is to be used amongst such people, well-designed studies need to be carried out. However, before such a study can commence, the availability of a glass-ionomer able to withstand the effect of the grinding of teeth, often observed in people with disabilities, is needed. Such a glass-ionomer should consist of properties like fast setting and high resistance against material fracturing and it needs to show high compressive and flexural strengths after initial setting. Such a restorative would not only be suitable for providing restorative care in people with disabilities but could also improve the survival rates of multiple-surface ART glass-ionomer restorations in primary and permanent teeth ${ }^{11}$, which are not currently meeting the American Dental Association (ADA) standard for unconditional use of dental materials ${ }^{20}$.

Several studies have assessed the mechanical properties of glass-ionomers currently used for ART procedures. The highest performances on flexural and compressive strengths for hand mixed formulas in vitro have been reported for the Fuji 9 (GC, Leuven, Belgium) and Ketac Molar Easymix (3M ESPE, Seefeld, Germany) $)^{3,5,14,18}$. In recent years, two encapsulated glass-ionomers for which the manufacturers claim high mechanical properties have been marketed. These are fastsetting high-viscosity glass-ionomer cements with a nanofilled resin coat (EQUIA system, GC, Tokyo, Japan) and a zinc-reinforced high-viscosity glassionomer (Chemfil Rock, Dentsply De-Trey, Konstanz,

\section{Germany).}

The aim of the present in-vitro study was to compare the diametral tensile (DTS), flexural (FS) and compressive (CS) strengths of the two encapsulated glass-ionomers with those of glassionomers currently in use. The null hypotheses tested were: there is no difference in each of the mechanical properties (diametral tensile, compressive and flexural strengths at the final breaking point) between: (1) The EQUIA system and glass-ionomers commonly used with ART; (2) The Chemfil Rock and glass-ionomers commonly used with ART; (3) The EQUIA system and Chemfil Rock.

\section{MATERIAL AND METHODS}

Approval to carry out this study was obtained from the ethical committee of the School of Dentistry, National University of Córdoba, Argentina (CIEIS) with reference number 18/2011. The four materials used in the present study are presented in Figure 1.

\section{Pilot studies and power calculation}

Because of the unavailability of information regarding the mechanical properties of the two developed encapsulated glass-ionomers, a pilot study was carried out in order to calculate the sample size required for the assessment of diametral tensile, flexural and compressive strengths. Based on these test results, 20 specimens of each test material were required for carrying out the DTS test,

\begin{tabular}{|c|c|c|c|c|c|}
\hline Product name & Manufacturer & Components & Batch no. & Expiry date & Shade \\
\hline \multirow[t]{2}{*}{ Fuji 9 Gold Label } & $\begin{array}{c}\text { GC Europe } \\
\text { (Leuven, Belgium) }\end{array}$ & $\begin{array}{l}\text { Powder: Fluoro-alumino-silicate glass, } \\
\text { polyacrylic acid powder }\end{array}$ & N219047 & 2013/11 & A3 \\
\hline & & $\begin{array}{l}\text { Liquid: Polyacrylic acid, polybasic } \\
\text { carboxylic acid }\end{array}$ & & & \\
\hline \multirow[t]{2}{*}{$\begin{array}{l}\text { Ketac Molar Easy } \\
\text { Mix }\end{array}$} & $\begin{array}{l}\text { 3M ESPE } \\
\text { (Seefeld, } \\
\text { Germany) }\end{array}$ & $\begin{array}{l}\text { Powder: Al-Ca-La fluorosilicate glass, } \\
5 \% \text { copolymer acid (acrylic and maleic } \\
\text { acid) }\end{array}$ & 406641 & $2016 / 06$ & A3 \\
\hline & & $\begin{array}{l}\text { Liquid: Polyalkenoic acid, tartaric acid, } \\
\text { water }\end{array}$ & & & \\
\hline \multirow[t]{2}{*}{ ChemFil Rock } & $\begin{array}{l}\text { Dentsply DeTrey } \\
\text { GmbH (Konstanz, } \\
\text { Germany) }\end{array}$ & $\begin{array}{l}\text { Zinc-modified fluoro-alumino-silicate } \\
\text { glass }\end{array}$ & 103000542 & $2014 / 02$ & A3 \\
\hline & & Polyacrylic and itaconic acid & & & \\
\hline \multirow[t]{2}{*}{$\begin{array}{l}\text { EQUIA system } \\
\text { (Fuji GP Extra + } \\
\text { G-Coat) }\end{array}$} & $\begin{array}{l}\text { GC Asia (Tokio, } \\
\text { Japan) }\end{array}$ & $\begin{array}{c}\text { Fuji } 9 \text { GP Extra: Water, fluoro-alumino- } \\
\text { silicate glass, polybasic carboxylic acid, } \\
\text { polyacrylic acid }\end{array}$ & 903039 & $2011 / 11$ & A3 \\
\hline & & $\begin{array}{l}\text { G-Coat: Methyl methacrylate, colloidal } \\
\text { silica, camphorquinone, urethane } \\
\text { methacrylate, phosphoric ester monomer }\end{array}$ & & & \\
\hline
\end{tabular}

Figure 1- Product name, manufacturer, composition, batch number, expiration date and shade of the materials tested 
60 for the FS test and 20 for the CS test.

\section{Specimen preparation for the DTS test}

Twenty specimens, $6.0 \mathrm{~mm}$ in diameter and 3.0 $\mathrm{mm}$ in height were prepared for each material. All the samples were made according to ADA Specification Number $66^{1}$. The environmental temperature was $23 \pm 2^{\circ} \mathrm{C}$ with a relative air humidity of $50 \pm 10 \%$ and the materials were mixed with a plastic instrument on an impermeable mixing paper. After mixing, the cement was put into plastic capsules and injected by a commercial syringe (Centrix, Shelton, CT, USA) into stainless steel moulds, which had previously been coated with vaseline ${ }^{\circledR}$. The moulds were slightly overfilled with the mixture over which a film of plastic was then placed. Finally, it was covered with a glass slide. Hand pressure was then applied for 20 seconds, extruding excess material from the top of the mould. Two minutes after the start of the mixing procedure, the assembly was placed in an oven at $37 \pm 1^{\circ} \mathrm{C}$ and $95 \pm 5 \%$ relative humidity for $15 \mathrm{~min}$. Then the specimens were ejected from the mould and stored in $6 \mathrm{ml}$ of distilled water at $37 \pm 1^{\circ} \mathrm{C}$ for 24 hours until tested. Before storage, specimens of the EQUIA system were coated with a nano-filled resin (G-Coat, GC Japan, Tokyo, Japan) and light-cured for 20 seconds on every surface with a LED device (Elipar Freelight 2, 3M ESPE, Seefeld, Germany).

\section{Specimen preparation for the FS test}

In accordance with the manufacturers' instructions for use, a total of 60 specimens of 25 $\mathrm{mm} \times 2 \mathrm{~mm} \times 2 \mathrm{~mm}$ bar-shaped dimensions for each of the four selected materials were prepared according to ISO 9917-2 Standards ${ }^{13}$ at room temperature $\left(23 \pm 2^{\circ} \mathrm{C}\right)$ by three trained operators, between 9:00 and 11:00 a.m. After 10 minutes of setting, the samples were removed from their moulds and immersed in paraffin at $37^{\circ} \mathrm{C}$ for 24 hours until tested. Before storage, specimens of the EQUIA system were coated with a nano-filled resin (G-Coat) and light-cured for 20 seconds on every surface with a LED device (Elipar Freelight 2, 3M ESPE, Seefeld, Germany).

\section{Specimen preparation for the CS test}

The four selected materials were tested in Class II ART-prepared cavities, using twenty extracted, non-carious, restoration-free human third molars each, stored in tap water at room temperature. Only those molars that ranged in size between 10.0 and $11.0 \mathrm{~mm}$ at the widest bucco-lingual dimension were included in the sample.

The preparation of the cavities was performed according to the description by Koenraads, et al. ${ }^{14}$ (2009). Two trained operators prepared the boxes to simulate class II ART cavities, using a spherical diamond bur (diameter 2.9 mm, 001-033 HP, Horico, Germany) in a high-speed, water-cooled handpiece. Finally, the cavity surface was smoothed with a medium-sized excavator (153/154, Henry Schein, USA). The exact dimensions and standard deviation (sd) of each resulting cavity were: $5.80(0.25) \mathrm{mm}$ for the largest bucco-lingual width; $4.00(0.25) \mathrm{mm}$ width for accessing the cavity from the occlusal surface; $2.90(0.25) \mathrm{mm}$ for the axial depth (mesio-distal) and an extension of the cavity to $1.00(0.25) \mathrm{mm}$ above the enamel-cement junction with an undermined enamel at the entire enameldentin junction of $0.5 \mathrm{~mm}$. Before the start of the restorative procedure, standardization of cavities was tested by means of polyether impressions (Impregum, 3M ESPE, Seefeld, Germany) and measured with sliding calipers. After application of a Tofflemire matrix band, all cavities were dried twice with a cotton pellet, conditioned with a moist cotton pellet dipped in the Fuji 9 liquid (GC, Leuven, Belgium) for 10 seconds and washed with cotton pellets dipped in distilled water for another 10 seconds and finally dried with dry cotton pellets. Restorative materials were applied to the cavities as described in the following sections and were stored in tap water at room temperature for three weeks before being tested, repeating exactly the same steps and conditions explained by Koenraads, et al. ${ }^{14}$ (2009).

\section{Hand mixed GIC's (Fuji 9 Gold Label and Ketac Molar Easymix)}

The powder and liquid were mixed according to the manufacturers' directions for use, until a homogeneous mixture was achieved within 30 seconds at room temperature $\left(21^{\circ} \mathrm{C}\right)$. The mixture was placed into the cavity in increments, using an applier/carver instrument (ART 102-6508; Henry Schein, USA) and an Ash 6 instrument (1052/6, Martin, Germany), and pushed into position with a medium-sized excavator (153/154, Henry Schein, USA). After placement of the final layer, a petroleum jelly-coated gloved finger held the glass-ionomer under pressure for 10 seconds. The excess material was removed with the applier/carver instrument and the medium-sized excavator. The restorative procedure was completed by placing a layer of petroleum jelly over the glass-ionomer to maintain the correct water balance within the restoratives.

\section{Encapsulated GICs (Chemfil Rock and EQUIA system)}

Each capsule of Fuji 9 GP Extra and Chemfil Rock was mixed for 10 seconds (Ventura Mix III, Madespa S.A., Madrid, Spain) and the mixture was immediately inserted into the cavity. After setting of the EQUIA cement, a layer of G-Coat (GC) was applied with a micro brush on the cement 
surface and light-cured for 20 seconds with an LED curing light (Elipar Freelight 2, 3M ESPE, Seefeld, Germany). The Chemfil Rock was condensed into the cavity under finger pressure after the finger was coated with a very thin layer of petroleum jelly.

\section{Testing mechanical properties}

Diametral Tensile Strength (DTS)

The diametral tensile strength test was performed after 1 day of storage. Tests were made in a Universal Testing Machine (Digimess MX5000, ARO S.A., Buenos Aires, Argentina) using a rounded rectangular testing rod at a crosshead speed of $0.5 \mathrm{~mm} / \mathrm{min}$. Only samples split into two pieces along their diameter after the breakdown point were considered for testing. The diametral tensile strength (DTS) $\left[\mathrm{kgf} / \mathrm{cm}^{2}\right]$ was calculated as follows: $\mathrm{DTS}=2 \mathrm{~L} / \mathrm{p} \mathrm{dh}$, where $\mathrm{L}$ is the load at fracture [kgf], $p=3.14, d$ is the diameter of the samples [0.6 $\mathrm{cm}$ ] and $\mathrm{h}$ is the height of the samples $[0.3 \mathrm{~cm}]$. DTS values $\left[\mathrm{kgf} / \mathrm{cm}^{2}\right]$ were converted into $\mathrm{MPa}$ as follows: DTS [MPa]=DTS [kgf/cm²] x 0.09807 .

\section{Flexural strength (FS)}

Before being tested, the specimens were measured with a digital micrometer to an accuracy of $0.01 \mathrm{~mm}$. Each specimen was subjected to a 3-point bending test on a universal testing machine (Digimess MX5000, ARO S.A., Buenos Aires, Argentina) with a rounded rectangular testing rod, at a crosshead speed of $1.0 \mathrm{~mm} / \mathrm{min}$. The GIC bars were supported by their extremes at a distance of $20.0 \mathrm{~mm}$ and loaded at their middle section until fracture occurred. The FS was calculated with the following equation: $F S=3 F I / 2 w h^{2}, F$ being the load at fracture, $I$ the distance between the two supporting points $(20.0 \mathrm{~mm})$ and $w h$ the width and height of the specimens $(2.0 \mathrm{~mm})$.

\section{Compressive strength (CS)}

Before being tested, all teeth were imbedded in an acrylic resin block. The coronal part of the tooth was exposed only in a direction perpendicular to the acrylic base. Samples were positioned in the testing machine as described by Koenraads, et al. ${ }^{14}$ (2009), forming a line on each resin block at an angle of $13.5^{\circ}$. Restorations were tested using a rounded rectangular testing rod applied on the marginal ridge at a crosshead speed of $1.0 \mathrm{~mm}$ / min until failure. In order to compare our results with those obtained by Koenraads, et al. ${ }^{14}$ (2009), values were expressed in Newton.

\section{Statistical analysis}

Data were analyzed by a biostatistician using SAS software. In order to compare the results with previous studies, the DTS and FS were calculated in megapascals (MPa), whereas CS was calculated in Newton. DTS, FS and CS were the dependent variables, while the different restorative glassionomer materials were the independent variables. ANOVA and Tukey B tests were applied to determine statistically significant differences between and within groups of restorative materials at an $a=0.05$, with a power of 0.9 one-sided.

\section{RESULTS}

The mean and standard deviations for the three strengths tests on the four brands of glassionomers are presented in Table 1. The EQUIA system showed the highest mean scores in all the three tests, followed by those of the Chemfil Rock, while the Fuji 9 Gold Label and Ketac Molar Easymix had the lowest mean test scores. Both the EQUIA system and Chemfil Rock had statistically significantly higher mean scores for all the three strength variables than the Fuji 9 Gold Label and Ketac Molar Easymix $(\alpha=0.05)$. The EQUIA had statistically significant higher mean scores for DTS and FS than the Chemfil Rock $(\alpha=0.05)$. Mean DTS, FS, CS values of the EQUIA system and Chemfil Rock were higher than those obtained with the Fuji 9 Gold Label and Ketac Molar Easymix; the difference varied between $11.2 \%$ and $72.3 \%$ (Table 2 ).

Table 1- Mean and standard deviation (SD) scores in MPa for diametral tensile strength (DTS), flexural strength (FS) and in Newton for compressive strength (CS) by the four brands of glass-ionomers

\begin{tabular}{|c|c|c|c|c|c|c|c|c|c|}
\hline & \multicolumn{3}{|c|}{ DTS } & \multicolumn{3}{|c|}{ FS } & \multicolumn{3}{|c|}{ cs } \\
\hline & $\mathbf{N}$ & mean & SD & $\mathbf{N}$ & mean & SD & $\mathbf{N}$ & mean & SD \\
\hline Ketac Molar Easymix & 20 & $7.5^{\mathrm{a}}$ & 0.7 & 60 & $28.9^{a}$ & 5.4 & 20 & $240.3^{a}$ & 37.7 \\
\hline Fuji 9 Gold Label & 20 & $7.7^{\mathrm{a}}$ & 0.5 & 60 & $41.8^{b}$ & 6.4 & 20 & $271.6^{a}$ & 52.2 \\
\hline Chemfil Rock & 20 & $9.4^{\mathrm{b}}$ & 0.7 & 60 & $46.5^{c}$ & 6.8 & 20 & $343.1^{\mathrm{b}}$ & 68.3 \\
\hline EQUIA & 20 & $10.0^{c}$ & 0.7 & 60 & $49.8^{d}$ & 6.4 & 20 & $358.5^{b}$ & 65.7 \\
\hline
\end{tabular}

$\mathrm{N}$, number of specimens

Different letters denote a statistically significant difference $(\alpha=0.05$; Tukey test) 
Table 2- Percentage increase in mean values for diametral tensile, flexural and compressive strengths between encapsulated and hand-mixed glass-ionomers tested in the present study

\begin{tabular}{lccc}
\hline \multicolumn{1}{c}{ Materials } & \% of increased DTS & \% of increased FS & \% of increased CS \\
\hline Chemfil Rock and Fuji 9 Gold Label & 23.3 & 11.2 & 26.5 \\
Chemfil Rock and Ketac Molar Easymix & 26.6 & 60.9 & 42.8 \\
EQUIA and Fuji 9 Gold Label & 29.8 & 19.1 & 32.1 \\
EQUIA and Ketac Molar Easymix & 33.3 & 72.3 & 49.1 \\
\hline
\end{tabular}

More than $30 \%$ of increased mechanical properties between the two materials is highlighted

Difference between the two materials in a greater than $30 \%$ increase in mechanical properties is highlighted

\section{DISCUSSION}

In order to test the aim of the present study, three different tests had been chosen. Specimens for testing flexural and diametral tensile strengths were prepared according to standardized specifications, while an original tooth-model that reproduced the situation of a Class II ART restoration ${ }^{14}$ was used in measuring the compressive strength. The purpose of the present study was not to determine the best adhesive restorative materials to be used with ART, but solely to compare the two encapsulated glassionomers with two glass-ionomers that have been extensively tested in other studies and are currently used with this approach. Therefore, a positive control group, e.g. a resin-based restorative, was not required in the study design.

The two null hypotheses were; that no difference in each of the physical-mechanical properties exists between the EQUIA system and the two commonly used glass-ionomers or between the Chemfil Rock and the same two glass-ionomers commonly used with ART, were rejected. The third null-hypothesis was partly rejected. Overall, the results showed that the two encapsulated glass-ionomers had significantly higher values for all three mechanical properties tested than the two hand-mixed glassionomers commonly used with ART.

The superior results for the mechanical properties of the two encapsulated glass-ionomers tested in the present study may be due to the additions of certain components. The coating agent added to the surface of the glass-ionomer of the EQUIA system contains a nanofilled resin that may have contributed significantly to the increased resistance of the material to mechanical forces. This assumption is supported by the results of an in vitro study which tested the influence of a resin coat (G-Coat Plus) spread over the Fuji 9 Extra and Ketac Molar Easymix ${ }^{4}$. Other studies have recommended that glass-ionomer surfaces, before being contaminated with water, should be coated in order to optimize their mechanical strengths ${ }^{15}$. The incorporation of reactive glass fillers modified with zinc oxide - which are easily released from the matrix - as well as the increment of itaconic acid in the liquid of Chemfil Rock, may explain the higher resistance of this new glass-ionomer when compared with that of the hand-mixed glass-ionomers, acting as network modifiers that increase reactivity of the powder and thus speeding maturation of the cement ${ }^{21}$. It is known that in the use of hand- mixed glass-ionomers, an extrinsic variability is introduced that may affect their performance ${ }^{6-8}$. This variability is dependent on the powder to liquid ratio and the number of porous elements or voids in the hand-mixed specimens and on the precision of the operator in inserting the mixture into the moulds and into the cavities. Use of encapsulated glass-ionomers nullifies the effect of that variability.

The Diametral Tensile Strength (DTS) test was selected as one of the three tests for the mechanical properties because it provides information related to the resistance of brittle materials such as conventional glass-ionomers. Specimens of the two hand-mixed glass-ionomers in the present study, prepared following standard specifications ADA \#66, resulted in mean DTS values that were in line with those obtained some years earlier for the same hand-mix glass-ionomers ${ }^{4}$. The results were also in line with values obtained for two other tested brands of glass-ionomer (Vitro Molar and Vitro Fil $)^{9}$. It was not possible to compare the DTS results for the EQUIA system and Chemfil Rock of the present study with those other studies.

The Compressive Strength (CS) test is often used to assess the ability of a material to withstand masticatory forces. A wide range of CS mean values (between 130 and $240 \mathrm{MPa}$ ) for the Fuji 9 and Ketac Molar have been reported after 24 hours of setting time, using standardized cylindrical specimens $s^{3,5,18,20}$. In the present study, an original model using Class II ART cavities for testing the materials was selected to simulate a clinical situation. Achieving the exact size and shape for each class II cavity, as well as the whole sequence of storage and testing of the specimens was challenging for the operators. A considerable number of samples had to be discarded since they were not as accurate as they should 
have been. Finally, the mean values obtained for the compressive strength (CS) of the Fuji 9 Gold Label and Ketac Molar Easymix in the present study were similar to those reported by Koenraads, et al. ${ }^{14}$ (2009). This method is different from the standard compressive strength tests that use cylindrical specimens. It means, therefore, that no comparisons with other published CS values can be made. We chose the Koenraads design because this model offered an in-vitro simulation of a restorative material subjected to a compressive load, as might happen clinically in a class II ART preparation. However, failure of the specimens in the tooth cavity system used was probably not due to pure compression but to a combination of compressive, tensile and shear stress failures instead.

The mean flexural strength (FS) values obtained in the present study were similar to those reported by Bonifácio, et al. ${ }^{3}$ (2009) for the Ketac Molar Easymix, but somewhat higher for the Fuji 9. According to the strong explanatory power found between the FS and long-term wear ${ }^{2}$, the authors suggested that it is possible to forecast the latter by using the FS test, which is considered to provide valuable information for the use of glass-ionomer materials in patients with strong grinding.

Considering the large difference in values for the three strength tests between the two new brands and the commonly used glass-ionomers, it appears that the EQUIA system and Chemfil Rock can be applied as a restorative material for treating dentine carious cavities in people with disabilities. Although a preliminary clinical study showed that the ART approach can function as a feasible strategy for controlling carious lesions in such populations ${ }^{16}$, introduction of the ART approach, using any of the two new brands of glass-ionomer in people with disabilities, would need to be closely monitored. If the EQUIA system and Chemfil Rock were also to be used for placing ART sealants in people with disabilities, the suitability of these two materials for this preventive measure would first need to be investigated. The final outcomes of the present study further indicate that these two encapsulated glass-ionomer materials may also contribute to an increase in the survival rates of multiple-surface ART glass-ionomer restorations in both primary and permanent teeth.

\section{CONCLUSIONS}

The two encapsulated high-viscosity glassionomers (EQUIA system and Chemfil Rock) had significantly higher test values for diametral tensile, flexural and compressive strengths than the commonly used hand-mixed high-viscosity glass-ionomers Fuji 9 Gold Label and Ketac Molar Easymix. The EQUIA system had significantly higher test values for diametral tensile and flexural strengths than the Chemfil Rock.

\section{ACKNOWLEDGMENTS}

Authors are very grateful to Dr. E. Bronkhorst for analyzing the data. We thank Dentsply, Germany; GC Europe, Belgium and 3M ESPE, Argentina for supplying the glass-ionomers. The study was financed by institutional funds from the SECYT, National University of Córdoba, Argentina.

\section{CONFLICT OF INTEREST}

Authors declare to have no conflict of interest

\section{REFERENCES}

1- American Dental Association. Specification no. 66 for dental glass ionomer cements. Council on Dental Materials, Instruments and Equipment. J Am Dent Assoc. 1989;119:205.

2- Amorim RG, Leal SC, Frencken JE. Survival of atraumatic restorative treatment (ART) sealants and restorations: a metaanalysis. Clin Oral Investig. 2012;16:429-41.

3- Bonifácio CC, Kleverlaan CJ, Raggio DP, Werner A, Carvalho RC, van Amerongen WE. Physical-mechanical properties of glass ionomer cements indicated for atraumatic restorative treatment. Aust Dent J. 2009;54:233-7.

4- Bonifácio CC, Werner A, Kleverlaan CJ. Coating glass-ionomer cements with a nanofilled resin. Acta Odontol Scand. 2012;70:4717.

5- Bresciani E, Barata TJ, Fagundes TC, Adachi A, Terrin MM, Navarro MF. Compressive and diametral tensile strengths of glass ionomer cements. J Appl Oral Sci. 2004;12(4):344-8.

6- Dowling $\mathrm{AH}$, Fleming $\mathrm{GJ}$. Is encapsulation of posterior glassionomer restoratives the solution to clinically induced variability introduced on mixing? Dent Mater. 2008;24:957-66.

7- Dowling $\mathrm{AH}$, Fleming GJ. Are encapsulated anterior glassionomer restoratives better than their hand-mixed equivalents? J Dent. 2009;37:133-40.

8- Esteves Barata TJ, Bresciani E, Cestari Fagundes T, Gigo Cefaly DF, Pereira Lauris JR, Lima Navarro MF. Fracture resistance of Class II glass-ionomer restorations. Am J Dent. 2008;21:163-7. 9- Fonseca RB, Branco CA, Quagliatto PS, Gonçalves LS, Soares CJ, Carlo $\mathrm{HL}$, et al. Influence of powder/liquid ratio on the radiodensity and diametral tensile strength of glass ionomer cements. J Appl Oral Sci. 2010;18:577-84.

10- Frencken JE. Evolution of the ART approach: highlights and achievements. J Appl Oral Sci. 2009;17:78-83.

11- Frencken JE, Leal SC, Navarro MF. Twenty-five-year atraumatic restorative treatment (ART) approach: a comprehensive overview. Clin Oral Invest. 2012;16:1337-46.

12- Holmgren CJ, Figueredo MC. Two decades of ART: improving on success through further research. J Appl Oral Sci. 2009;17:122-33. 13- International Organization for Standardization. ISO $9917-$ 2:2010: Dentistry. Water-based cements. Geneva: ISO; 2010. 14- Koenraads H, van der Kroon G, Frencken JE. Compressive strength of two newly developed glass-ionomer materials for use with the Atraumatic Restorative Treatment (ART) approach in class II cavities. Dent Mater. 2009;25:551-6.

15- Lohbauer U, Krämer N, Siedschlag G, Schubert EW, Lauerer $B$, Müller FA, et al. Strength and wear resistance of a dental glass-ionomer cement with a novel nanofilled resin coating. Am J Dent. 2011;24:124-8.

16- Molina G, Kultje C. Atraumatic Restorative Treatment with Carisolv in intellectually disabled patients. 1 year follow-up. J Dis Oral HIth. 2003;4:15-8. 
17- Molina GF, Leal SC, Frencken JE. Strategies for managing carious lesions in patients with disabilities - a systematic review. J Dis Oral HIth. 2011;12:157-69.

18- Peez R, Frank S. The physical-mechanical performance of the new Ketac Molar Easymix compared to commercially available glass ionomer restoratives. J Dent. 2006;34:582-7.

19- Wyatt CC, MacEntee MI. Dental caries in chronically disabled elders. Spec Care Dent. 1997;17:196-202.
20- Yip HK, Smales RJ, Ngo HC, Tay FR, Chu FC. Selection of restorative materials for the atraumatic restorative treatment (ART) approach: a review. Spec Care Dentist. 2001;21:216-21. 21-Zoergiebel J, Ilie N. Evaluation of a conventional glass ionomer cement with new zinc formulation: effect of coating, aging and storage agents. Clin Oral Investig. 2013;17:619-26. 